COMPTES RENDUS

\title{
HISTOIRE ÉCONOMIQUE
}

La Forêt. Actes du $113^{\mathrm{e}}$ Congrès national des sociétés savantes, Strasbourg, 1988. Paris, Éd. du Comité des travaux historiques et scientifiques, 1991. 16 x 24, 380 p. ("Colloques du C.T.H.S. ", 6).

Le Bois et la ville. Du Moyen Age au $x^{2}$ siècle. Actes du Colloque de Saint-Cloud, 18-19 nov. 1988, éd. par Jean-Louis Biget, Jean Boissière et Jean-Claude Hervé, introd. par Daniel Roche. Fontenay/Saint-Cloud, École normale supérieure, 1991. 17,3 x 24,5, 318 p. (« Cahiers de Fontenay »).

Une heureuse coïncidence nous offre la publication simultanée de deux colloques très réussis, qui sont proches l'un de l'autre à la fois par leurs thèmes et surtout par l'état d'esprit dans lequel ils ont été conçus. Tous deux, en effet, ont trait à la gestion des forêts, à l'exploitation du bois, à son transport, à son utilisation, voire à sa symbolique. Tous deux sont riches de la confrontation de différents points de vue, ceux des géographes et des historiens, bien sûr, mais aussi des agronomes ou des sociologues. Tous deux, enfin, ont pris pour cadre une très longue durée, ce qui permet, au fil des contributions, d'évaluer à leur juste mesure continuités et mutations.

Les actes du colloque consacré à la forêt, qui s'est tenu à Strasbourg en 1988, s'ouvrent par une belle étude de P. Arnould : il montre que définir une forêt, même de nos jours, ne va pas de soi. Malgré le perfectionnement des instruments et des techniques d'arpentage, dont il fait l'historique depuis Colbert jusqu'à la télédétection, les évaluations de la superficie forestière mondiale actuelle restent très divergentes - entre 2,4 et 6 milliards d'hectares - ce qui prouve qu'à l'évidence, tous les chercheurs «ne mesurent pas la même réalité ». Les trois communications suivantes étudient les problèmes particuliers qui se posent à quelques grands types de forêts et prolongent bien cette analyse : celle de la côte aquitaine, protégée par une dune artificielle et calibrée sur $230 \mathrm{~km}$ de long, n'est pas soumise aux mêmes contraintes que la forêt de Haye, essentiellement marquée par sa proximité avec la ville de Nancy. De même, les études qui traitent des menaces sur la forêt (parasites, incendies) ou de son aménagement font une large place aux phénomènes locaux, aux paramètres bien spécifiques qui affectent la couverture forestière de tel ou tel « pays"

La seconde partie du colloque, consacrée à l'histoire de la forêt, rassemble quatorze communications classées par ordre chronologique. Dans la partie consacrée 
à " la forêt des temps lointains $», \mathrm{~J}$-C. Koeniguer, en particulier, fait une solide et très utile synthèse de nos connaissances actuelles sur la forêt en Europe occidentale et dans le Bassin méditerranéen du $v^{\varepsilon}$ au $\mathrm{I}^{\mathrm{er}}$ millénaire. Préférant plutôt approfondir un cas particulier, R. Fossier reprend un dossier qui avait déjà attiré son attention quelques décennies plus tôt : le défrichement de la Vicogne, en Picardie. En combinant l'examen des rares documents sur le sujet et l'analyse rigoureuse de la toponymie locale, il parvient ainsi à en restituer la chronologie, même si les moyens mis en œuvre ici par les hommes restent mal connus, faute de textes suffisamment loquaces.

À l'époque moderne, des sources de plus en plus riches et variées traduisent des préoccupations nouvelles quant à la gestion des ressources sylvicoles. G. Plaisance décrit ainsi une grande enquête, qui fut menée en Bourgogne dès 1583, pour connaître l'étendue des dégradations dues à la croissance de la population et à l'absence de toute surveillance efficace. Ce constat fut immédiatement suivi d'une réglementation qui, par sa précision, annonçait avec près d'un siècle d'avance la politique de Colbert. Menée par trois commissaires nommés par le roi, cette " Réformation " prouve donc que le souverain accordait une attention toute particulière à la forêt de son domaine dès la fin du Xvie siècle. Dans le Quillanais, C. Fruhauf observe une préoccupation similaire, même si les carences des officiers locaux chargés des Eaux et Forêts ont ici parfois tendance à la priver d'une efficacité réelle. Au xviri siècle, l'État semble désormais s'être doté des outils nécessaires à un meilleur contrôle de ses forêts. Ainsi, la belle communication de F. Vion-Delphin prend comme exemple la gruerie de Pontarlier, où l'on voít une administration bien rôdée se mettre en place progressivement, après l'annexion de la Franche-Comté en 1678. Mais ce renforcement de la surveillance, qui tente de réprimer les coupes sauvages, ne permet pas d'éviter une très grave crise de l'approvisionnement en bois dans cette région à la fin de l'Ancien Régime.

$\mathrm{Au} \mathrm{XIX}^{\mathrm{e}}$ siècle, la forêt est de plus en plus prise en compte par tous ceux qui réfléchissent aux problèmes posés par l'aménagement du territoire. $\mathrm{B}$. Kalaora et A. Savoye distinguent alors deux conceptions radicalement différentes : d'une part, une pratique d'aménagement assez autoritaire, abstraite et universaliste, très nette sous le Second Empire, et d'autre part, une politique plus souple, élaboré par F. Le Play et son école, qui domine dans les premières décennies de la III ${ }^{e}$ République, et qui prend d'abord en compte les besoins des populations locales, pour associer le mieux possible agriculture et sylviculture. Malgré leurs divergences, ces deux politiques tentaient néanmoins de contribuer par le reboisement au développement économique et social des régions défavorisées. Ce fut notamment le cas dans le Dauphiné, étudié ici par P. Chevalier et M.J. Couailhac.

Le colloque organisé par le Centre d'histoire urbaine de l'École normale supérieure de Fontenay/Saint-Cloud est davantage centré sur les liens d'interdépendance qui se sont tissés depuis le Moyen Àge entre le bois et la ville : l'approvisionnement et la consommation en bois de chauffage, les transformations de la forêt sous la pression citadine, l'utilisation du bois dans la construction et, enfin, son impact social, économique et symbolique dans la ville. Toutes les communications consacrées au xvir ${ }^{e}$ siècle insistent sur le fait que le ravitaillement des 
villes en bois de chauffage posait aux autorités des problèmes tout aussi redoutables que l'approvisionnement en grains. À partir de l'exemple de la Lorraine, J.-P. Husson montre qu'à la fin du siècle, les difficultés ont même tendance à s'accentuer du fait de la croissance urbaine, des déprédations causées par les droits d'usage des communautés rurales et de l'essor pré-industriel régional. En Franche-Comté, F. Vion-Delphin dresse un constat assez semblable et décrit avec une grande précision les mesures qui sont édictées pour alimenter avec plus de régularité le marché au bois de Besançon. Quelques particuliers tentent aussi, à leur manière, de répondre à cet essor de la consommation, où ils voient une importante source de profits : c'est le cas, par exemple, du duc de Bouillon, qui est à l'origine en 1725 d'un projet de canal qui aurait permis d'écouler ses bois d'Evreux à Paris et à Rouen.

Avec les $\mathrm{XIX}^{e}$ et $\mathrm{xx}^{e}$ siècles, les rapports entre la ville et les forêts péri-urbaines se modifient sensiblement. A. Bloch observe qu'autour de Strasbourg, dès le Second Empire, l'exploitation du bois doit se combiner de plus en plus avec la sauvegarde ou la reconstruction artificielle d'un massif forestier désormais conçu comme un lieu de détente. B. Kalaora décèle une évolution similaire en forêt de Fontainebleau, où les élites parisiennes viennent par le nouveau chemin de fer se promener dans ce qu'elles considèrent comme un «musée naturel ». La forêt devient alors, insensiblement, un argument de vente et un pôle d'attraction pour les lotissements pavillonnaires, à condition toutefois qu'elle n'en soit pas trop proche ; c'est ce que montre A. Fourcault dans un travail remarquablement documenté sur Montmorency dans les années 1920.

La place du bois dans la conception des maisons anciennes est étudiée en particulier par B. Toulier et J.-M. Pérouse de Montclos. Le premier se livre à une savante description des maisons à pans de bois construites dans le Val de Loire et le Berry aux $x v^{e}$ et $x v^{e}$ siècles. Le second étudie les innovations techniques en matière de charpente, en les mettant en relation avec la pénurie de grands bois qui affecte la construction urbaine tout au long de l'époque moderne.

Enfin, l'importance sociale du bois est abordée dans les dernières communications. S. Roux décrit la diversité des métiers du bois à Paris du XIII au Xv' siècle et estime qu'ils donnent du travail à 7 ou $10 \%$ de la population parisienne selon les époques - soit moins que l'alimentation, mais autant que le textile. Quant à la symbolique du bois, elle est explorée au Moyen Âge par M. Pastoureau, qui insiste notamment sur la noblesse du matériau (celui de la Croix) et l'image positive du charpentier (le métier de Joseph).

Du fait de leur richesse et de leur diversité, de l'importance qu'ils accordent à la longue durée, de la pluridisciplinarité des participants, ces deux colloques sont désormais des ouvrages de référence pour tout un champ de la recherche. Ils ne devraient pas manquer de susciter d'autres initiatives, visant à mieux comprendre un domaine encore méconnu. 
Forges et forêts. Recherches sur la consommation proto-industrielle de bois. Publié sous la dir. de Denis Woronoff. Paris, Éd. de l'École des hautes études en sciences sociales, 1990. $15 \times 22,5,263$ p., ill., graph., cartes ( $\ll$ Recherches d'histoire et de sciences sociales $\%, 43$ ).

Dix historiens de l'aménagement forestier ou de la proto-industrie ont uni leurs contributions pour esquisser une fresque de la lutte contre les pénuries de combustible ligneux, depuis le Moyen Âge classique jusqu'à la veille de 1914. Au centre de cette description, apparaissent les fourneaux sidérurgiques et les forges, installations longtemps accusées par la rumeur publique de tarir les approvisionnements des foyers domestiques.

En ce sens, l'activité métallurgique est tenue pour responsable de froidures que doivent endurer les pauvres : elle semble érigée en bouc émissaire lors des flambées du prix des bois, subissant le même opprobre que les marchands de grains, lorsque ceux-ci sont visés par les dénonciateurs du "pacte de famine".

Dès ses premières pages, l'ouvrage consacré aux Forges et forêts se démarque de ce cliché polémique et pamphlétaire, en démontant les constantes qui poussent les Cahiers de doléances de 1789 à reprocher aux sidérurgistes de "dévorer le bois des familles $\%$. D'ailleurs, dans tout le livre, les effets dévastateurs de la consommation familiale sont constamment évoqués en creux, avec une invitation à multiplier les recherches sur cette question. Avec la repopulation du XvI siècle, il apparait ainsi que le prélèvement populaire sur la forêt bretonne encourage plutôt la lande agro-pastorale que la conservation équilibrée des bois au sein des terroirs.

Inversement, Forges et forêts souligne que, tout en consommant autant que des villes voisines, l'activité sidérurgique innove en induisant la nécessité d'une économie forestière calculée, done d'une réflexion qui cesse de considérer le bois comme un lieu de prélèvement prédateur, pour y créer la possibilité d'un renouvellement rationnel des ressources.

Si la notion d'aménagement territorial est largement issue du compartimentage technique des gisements forestiers, la volonté d'adapter le prođuit sylvestre à des débouchés diversifiés, tout en épargnant son potentiel de reproduction, est contemporaine de la naissance de l'économie politique. C'est, en effet, all cours du second $x v^{\mathrm{e}}$ siècle, à l'époque de Jean Bodin, que la nécessité d'encadrer au plus juste l'exploitation forestière se fait sentir. C'est aussi le moment où le pouvoir royal multiplie à l'envi les offices de forestiers, les revenus de la forêt supposant toujours une dimension fiscale.

Quel rôle la sidérurgie tient-elle dans ce resserrement de la surveillance administrative et de la gestion forestière? Une part prépondérante, d'après les recherches évoquées dans Forges et forêts. À la fin du XIII siècle, le développement des bas fourneaux provoque sans doute localement des surcharges dans la consommation forestière, en un mouvement qui s'apparente à la saturation que connaissent alors les terroirs, au plan des disponibilités alimentaires.

Avec la grande peste de 1348-1349, et la catastrophe démographique qui en résulte, la forêt regagne sur les défrichements, tandis que l'utilisation du bois diminue : dans cette ambiance d'abondance est introduite en France la rêduction 
indirecte, qui dédouble les feux métallurgiques par le biais des hauts fourneaux et des forges d'affinage, les secondes transformant en fer la fonte des premiers.

Avec la fin du «beau xvi siècle», vers 1550 , les tensions sur l'approvisionnement en bois de feu réapparaissent. Dès lors, se dessine une longue période de reconversion des forêts, menée dans le but d'adapter les cycles végétatifs de l'arbre à l'essor sidérurgique, dont l'importance stratégique n'échappe pas à l'autorité royale.

C'est ainsi que, de 1550 à 1720 , les forêts sont progressivement aménagées, suivant trois orientations directrices : le cantonnement avec réduction des surfaces ouvertes aux droits d'usage, la mise en coupe réglée et l'accélération des rotations avec prime au taillis. L'ordonnance colbertienne de 1669 doit de la sorte être comprise comme un essai de codification pour une évolution déjà bien engagée.

Cette mise en ordre juridique, qui vise à maintenir un équilibre entre les consommateurs de bois, est fortement dérangée par la criante pénurie des années 1720. Un palliatif est néanmoins fourni par les Lumières, avec les recherches de savants comme Réaumur ou Buffon. Dès lors, les académies provinciales contribuent à déplacer le souci d'épargne de la sylviculture vers les opérations industrielles : elles prônent l'amélioration des meules de charbon, le développement des hauts fourneaux à section circulaire, le voûtage des foyers de forge, ou l'emploi de la méthode comtoise, qui réunit sur un seul feu la fonderie et l'affinage.

Voux pieux jusqu'en 1780, ces solutions sont massivement utilisées après 1800, les négligences révolutionnaires ayant semé l'alarme dans les forêts, tandis que les arguments de chimistes comme Lavoisier ou Berthollet faisaient école. Pourtant, les savants de la Révolution éprouvent le sentiment d'une demi-victoire en matière de combustible sidérurgique : ils échouent dans leur volonté d'introduire la houille dans les hauts foumeaux, en particulier à cause du retard pris par la France en matière de communications fluviales.

Quoi qu'il en soit, les canaux de la Restauration favorisent l'emploi de la houille dans les forges, libérant une plage de consommation pour les hauts fourneaux qui se multiplient et, particularité française, sont toujours chauffés au bois. Alors même que les Eaux et Forêts plaident pour le bois d'œuvre et que le corps des Mines préconise la fonte au charbon de terre, la technique française s'efforce d'accroître la productivité sans renoncer aux capacités énergétiques de la forêt : elle tente de mêler du bois vert ou torréfié au charbon de bois, elle essaie de réchauffer les fours avec les gaz perdus et elle recommande le combustible mixte, à la fois minéral et végétal.

C'est sous le signe de tels compromis inventifs que se produit le premier essor sidérurgique de la France industrielle, entre 1815 et $1850:$ il facilite en aval la construction des premiers chemins de fer. Après 1860, le développement ferroviaire encourage en retour la généralisation du tout à la houille : tandis que les Eaux et Forêts font progresser les futaies, la consommation urbaine pousse le bois de chauffage à la hausse, et les trains distribuent aux métallurgistes une houille de substitution, provenant notamment du Pas-de-Calais. Les usines sidérurgiques trop à l'écart sont à terme condamnées : la période 1880-1920 est fatale aux forges qui conservent le bois. 
Au terme d'une évolution de plus de trois siècles, les besoins des forges ont laissé leur marque sur le boisement: plusieurs pages de Forges et forêts prêtent attention à l'archéologie paysagère.

Au nord-est de la France, la volonté de fixer des populations sur les frontières se marque ainsi par l'abondance des communaux, dont les coupes sont vendues aux sidérurgistes du XIX siècle. Dans l'ouest au contraire, l'affouage sidérurgique a préservé les résidus forestiers de la mise en landes par les villageois. Au sud-est s'opposent les fonds de vallée, ressemés pour les besoins de la sidérurgie, et les forêts de versants, plus largement laissées à la génération spontanée. Dans les Pyrénées, les hêtraies ariégeoises portent la marque des tolérances consenties aux sidénurgistes, tandis que le bassin versant de l'Aude est plus enrésiné, l'administration royale y ayant avantagé le bois d'cuvre.

Par sa double dimension historique et géographique, Forges et forêts met en scène les mécanismes humains qui produisent les formations paysagères. À l'heure où le législateur se préoccupe du gaspillage de l'espace, mesurant la nécessité de réguler l'aménagement pour composer des paysages, ce livre contribue à éclairer historiquement un enjeu d'actualité.

Jean-Marcel GoGer.

Bronislaw Geremek, Les Fils de Caïn. L'image des pauvres et des vagabonds dans la littérature européenne du $x \mathscr{V}^{f}$ au $x и f^{2}$ siècle. Av.-pr. trad. de l'italien par Françoise Pineau, texte et notes trad. du polonais par Joanna ArnoldMoricet. Paris, Flammarion, 1991. 15 x 24, 418 p., index.

Il n'est plus nécessaire de présenter Bronislaw Geremek, l'historien des pauvres de l'Europe médiévale et moderne. Saluons avant tout l'originalité de son étude qui concilie ou plutôt réconcilie histoire et littérature en faisant de la seconde non pas une auxiliaire pour la première, mais une source à part entière. Cette approche, quelque peu délaissée en Europe occidentale, correspond en Pologne à une longue tradition de travaux qui ont utilisé une documentation littéraire, faute d'archives disparues dans la tourmente des guerres qui n'a pas épargné la patrie de Bronislaw Geremek. Les Fils de Cain ont aussi une histoire : interdits de publication en Pologne, en 1980, en raison de l'engagement de premier plan de leur auteur au sein du syndicat Solidarité, ils ont été édités pour la première fois en italien, en 1988, soit trois ans avant la présente édition française. Ces difficultés de parution se ressentent dans le nombre des traducteurs - cinq au total - et dans l'inégale qualité de leurs traductions d'un chapitre à l'autre ou à l'intérieur d'un même chapitre. Mais le résultat est incontestable : cet ouvrage, tel un sésame, contribue à éclairer des ceuvres devenues chefs d'wuvre, qui gagnent encore à être confrontées et comparées, avec pour trame européenne les multiples visages de la pauvreté et les figures tellement diverses de pauvres désormais catalogués pour des raisons de répression. Parfait connaisseur de ce milieu régi par d'autres lois, d'autres formes de gouvernement, d'autres usages et, surtout, d'autres langues, 
Bronislaw Geremek montre comment la société des pauvres alimente la littérature du temps, qu'elle soit allemande, anglaise, italienne, française et espagnole. Certes, chaque pays a sa spécificité : l'Allemagne de Simplex Simplicissimus et de la vagabonde Courage, au sortir de la terrible guerre de Trente Ans, ne saurait se confondre avec les bas-fonds de l'Angleterre élisabéthaine qui ont inspiré Shakespeare et alimenté pour des siècles les experts en tricheries et escroqueries de tout genre. La France n'est pas en reste, même si elle accuse un léger retard par rapport à l'Angleterre et à l'Espagne dans la description du « milieu » des mendiants et des vagabonds. Au xuII siècle, circule dans les années 1660 la volumineuse $H i s-$ toire et recherches des Antiquités de Paris d'Henri Sauval qui ne paraitra qu'en 1724. Victor Hugo s'en inspire beaucoup dans Notre-Dame de Paris après avoir transposé à la fin du Moyen Âge les réalités parisiennes de la cour des Miracles du xvir siècle, ce même siècle où se met en place le "grand enfermement". Mais la rencontre majeure entre marginalité et bonne société se fait dans l'Espagne au Siècle d'or avec l'épanouissement de la littérature picaresque et de son anti-héros, le picaro. Autant de pays et de grandes villes, autant de personnages qui ont en commun d'être hors société et de faire éprouver à ceux qui les observent et les décrivent autant de crainte que d'attrait : ainsi se précise une "cosmographie de l'antisociété ", où Bronislaw Geremek guide ses lecteurs, pour leur plus grand plaisir.

Anne-Marie Cocula.

Daniel Roche, La Culture des apparences. Une histoire du vêtement (xvI ${ }^{e}-x \eta u I^{e}$ siècle). Paris, Fayard, 1989. $14 \times 21,549$ p., index.

"L'habit ne fait pas le moine." Pourtant... Servons-nous de ce proverbe sésame pour entrer de plain-pied dans le bel ouvrage de Daniel Roche. Ce livre est à la fois le résultat d'une longue réflexion, d'une connaissance sensible et scientifique de l'Ancien Régime et d'une collaboration fructueuse avec des étudiants qui ont apporté leurs gerbes à cette moisson. Car tout fait ventre dans la quête menée par l'historien pour saisir, définir, apprécier une «culture des apparences " aussi ancienne peut-être que le péché originel : « Et ils connurent qu'ils étaient nus. " Cet extrait de la Genèse, placé en tête du chapitre I, est une nécessaire entrée en matière.

Avec le temps, les descendants d'Adam et Ève surent protéger leur nudité, la camoufler sous d'amples habits, avant d'apprendre à la suggérer ou à l'extraire lentement de vêtements dont l'histoire devient témoignage et symbole d'une culture, celle des « apparences ». Le sous-titre de l'ouvrage, Une histoire du vêtement ( $x \cdots f^{f}-x h I^{\prime}$ siecle), indique clairement la piste jalonnée et parcourue. La période choisie est au carrefour de sources complémentaires dont les plus riches, les inventaires après décès, font pénétrer le lecteur dans l'intimité des Français d'autrefois. Chercheur rigoureux et curieux, D. Roche a ouvert les portes de leurs armoires, inspecté les étagères, vidé les tìroirs, sans rien négliger des composantes 
de garde-robes de toutes origines, répertoriées, analysées et comparées en 1700 et 1789.

Ce bilan du siècle des Lumières révèle des changements déjà révolutionnaires, comme l'éclosion vestimentaire d'une féminité épanouie ou «l'invention du linge " et sa diffusion par l'intermédiaire de la noblesse et de sa domesticité. Deux mondes à la fois si proches et si distants! Laquais et valets, servantes et lingères, habillés selon le goût de leurs maitres et maitresses, ont été les mannequins d'une mode qui parcourt aisément la distance de Versailles à Paris, pénètre dans le peuple et progresse en province avec des écarts et des retards significatifs. Ces transformations ont des limites. Ainsi caleçons et culottes, le linge le plus près du corps, tardent à s'imposer comme si les lointains enfants d'Adam et Ėve n'étaient pas parvenus encore au camouflage idéal de leur nudité. Il faut attendre le $\mathrm{XIX}^{\mathrm{e}}$ siècle pour que "le linge invisible " progresse en même temps que la propreté.

Ainsi, le costume s'intègre dans un système de valeurs religieuses, morales et politiques, et devient signe d'appartenance sociale avec pour références la Cour et la Ville. Pour saisir ressemblances et différences à partir de ces modèles, il convient de questionner les livres de comptes des privilégiés où se mesure selon les familles, leur fortune et leur résidence, la contagion vestimentaire avec les Grands de Versailles. Bien plus difficile, en raison de la rareté des sources, est l'estimation des dépenses vestimentaires du peuple au moment où il succombe lui aussi à la tentation de la consommation.

Tentation : tel est peut-être le maître-mot de la "distribution des apparences " qui fait l'objet de la troisième partie de l'ouvrage. Fabrication et production restent encore tributaires de tout un réseau d'artisans, de marchands et de boutiquiers exposés aux rigueurs d'une réglementation mal adaptée aux changements. Dès lors, se développent les trafics parallèles et défendus des vêtements volés et revendus. Tandis qu'en marge se ramifient les circuits de la friperie, étonnant dédale de boutiques et de pratiques que $\mathrm{D}$. Roche décrit avec une certaine jubilation afin de mettre en valeur une «culture fripière ", parisienne et populaire, où s'approvisionnent les clients les plus divers.

Précieux, convoités, mieux diffusés, marque d'une appartenance sociale, ces vêtements deviennent l'objet de soins nécessaires à leur entretien et à leur longévité. Là encore, dans la plupart des milieux, le siècle des Lumières révèle des transformations décisives où l'humble blanchisseuse a sa place aux côtés de l'utopie lavandière d'un Goyon de la Plombanie...

Restent la part du concret et l'appel à un imaginaire doublé de réalité : saluons dans cet ouvrage la place accordée à une illustration commentée - hélas ! en noir et blanc - , le recours au contenu des romans, témoins des sollicitations et des aventures des habits de toute sorte, et l'utilisation du message des gravures, celles de la mode et celles de l'Encyclopédie. Voilà que la culture des apparences, dévoilée dans cet ouvrage, cesse de l'être pour devenir culture et que la mode se dépouille de l'éphémère pour s'installer dans la longue durée de l'histoire des hommes. 
François Hincker, La Révolution française et l'économie. Décollage ou catastrophe? Paris, Nathan, 1989. 13 x 19, 224 p., bibliogr., index (« Circa. Économie Sciences sociales $m$ ).

La Révolution française a-t-elle été une "catastrophe nationale " au plan économique, comme le prétend Maurice Lévy-Leboyer? A-t-elle fait « dégringoler la France et l'Europe continentale de la ligne de crête de l'évolution », comme l'en accuse Pierre Chaunu? Autant d'affirmations péremptoires qui n'invitent guère à la sérénité. L'histoire économique de la Révolution se trouve ainsi grevee d'enjeux idéologiques, aussi vieux que la Révolution elle-même, qui concernent l'appréciation de ses effets : dès l'époque du Consulat et de l'Empire, ses partisans et adversaires avaient fait du bilan économique de la décennie 1789-1799 un enjeu politique; et les historiens ont emboîté le pas.

Or, jusqu'à récemment, les travaux étaient rares, qui auraient permis d'étayer sérieusement un bilan complet. En effet, si c'est une heureuse tradition française que de pratiquer une histoire à la fois économique et sociale, il apparait que, concernant la Révolution, le premier terme a en fait été largement négligé au profit du second. D'autre part, les historiens ont longtemps privilégié l'étude des phénomènes exceptionnels introduits par l'événement révolutionnaire, comme la vente des biens nationaux, ou l'adoption du Maximum des prix; réciproquement, le marché foncier " normal " et l'histoire proprement dite des prix étaient négligés, accréditant l'idée d'un dérèglement général de l'économie, d'une tourmente brisant tous les mécanismes anciens : les thèses "catastrophistes" en étaient confortées.

Mais depuis une dizaine d'années, notre connaissance proprement économique de la période révolutionnaire et impériale a largement progressé. François Hincker en rassemble les résultats les plus récents dans une mise au point claire et nuancée, de présentation très agréable.

L'ouvrage balaie tout d'abord quelques idées reçues et fausses évidences : la Révolution n'a pas été la Saint-Barthélemy des entrepreneurs, les entreprises ont survécu; loin d'être des apprentis sorciers de l'économie, les révolutionnaires disposaient d'une sérieuse culture en la matière, ainsi que l'attestent discours et débats des assemblées. Surtout, c'est à tort que certains ont voulu voir, dans les mesures de l'an II, l'essence même d'un projet dirigiste utopique, car les hommes de la Convention étaient bien des libéraux, respectueux de la propriété et convaincus que l'ordre social doit tendre à la liberté des échanges : la guerre seule, et ses circonstances exceptionnelles, expliquent qu'ils aient cédé à la pression sansculotte en faveur du Maximum et des réquisitions. Mais il est vrai que leur libéralisme, tempéré par la notion d'utilité publique, s'accommode fort bien d'une intervention économique de l'État : celle qui créera les conditions concrètes d'un accès égal de tous au marché, guidant vers la liberté économique une nation qui n'y est pas accoutumée. Pour être moins optimistes que Smith, ils n'en sont pas moins sincèrement libéraux. Il est sûr, enfin, que l'expérience de l'assignat fut un échec : F. Hincker décrit les dérives et glissements successifs qui conduisent à l'hyperinflation. Mais loin d'être un destin, cet engrenage a des causes multiples, 
dont l'analyse peut rendre raison. Du reste, il n'était pas stupide de chercher le moyen d'organiser un système de crédit public pour faire face à la crise financière léguée par la monarchie. Avec le recul, et quelque cynisme, on peut même, expost, trouver une grande vertu à la catastrophe monétaire : « Cette formidable inflation suivie d'une non moins formidable deflation fonctionna comme une purge ", provoquant un désendettement général. Cet incroyable assainissement financier, au total, ruina le rentier, mais permit au paysan et à l'entrepreneur d'investir à bon compte, qui dans la terre, qui dans des bâtiments.

Le bilan, dressé avec méthode et prudence, ne peut qu'être contrasté. L'empire colonial est perdu; mais sa richesse était trompeuse : il est douteux qu'il ait pu, à long terme, soutenir une industrialisation métropolitaine, comme ce fut le cas pour l'Angleterre. La perte des colonies a entrainé le déclin de la façade atlantique, au profit de la France continentale et de Paris. Les paysans sont les autres grands bénéficiaires de la Révolution. La propriété s'en est trouvée morcelée, et l'exode rural ralenti du même coup. Mais Georges Lefebvre appréciait positivement, du point de vue de la démocratie politique, qu'ait été épargnée à la France la sauvagerie d'un capitalisme débridé à l'anglaise (expulsion des petits paysans par les enclosures, et prolétarisation urbaine massive). De plus, il apparait aujourd'hui que la petite propriété foncière n'a nullement démérité en matière de rendements et de productivité de la terre. Bien au contraire, pour F. Hincker, c'est la grande propriété qui fait problème : entendons l'attrait qu'exercent la rente foncière et le style de vie aristocratique sur les élites bourgeoises. Véritable revanche posthume de l'Ancien Régime, l'engouement pour le placement foncier détoume de l'investissement productif et du placement mobilier une bonne part des profits industriels et commerciaux. Le développement des activités capitalistes se trouve ainsi ralenti, au début du XXx siècle, par le manque de capitaux et la faiblesse relative du marché financier. Mais c'est la continuité avec l'Ancien Régime qui est en cause ici, et non la rupture révolutionnaire.

D'où la conclusion très nette de l'auteur : révolution propriétaire assurément, la Révolution française n'est pas la " révolution capitaliste », « libératrice des forces productives ", que certains ont prétendu, s'autorisant abusivement de Marx. Industriels et négociants, d'ailleurs, n'y furent pas aux avant-postes, et c'est la "classe propriétaire " qui imposa ses valeurs : terre, rente, épargne, plutôt que profit et investissement.

Philippe Munard.

Villes, bonnes villes, cirés et capitales ; études d'histoire urbaine (xI'-xVII' siècle) offertes à Bernard Chevalier. Éd. Monique Bourin. Tours, Publications de l'Université de Tours, 1989. 16 × 24, VII-419 p.

Les mélanges offerts à Bernard Chevalier rassemblent 39 études, 38 d'histoire urbaine et 1 essai de J.-P. Genet de Paris-I ( "Féodalisme et naissance de l'État moderne : à propos des thèses de Charles Tilly », p. 239-246) couvrant une pé- 
riode allant du XI $I^{\mathrm{e}}$ au XVII ${ }^{\mathrm{e}}$ siècle (et non du XII au XVIII' siècle comme l'annonce le sous-titre). La richesse du volume est grande - entendons sa richesse intellectuelle, car sa réalisation matérielle est médiocre. Si 27 communications portent sur le Moyen Âge, 12 portent sur les Temps modernes et, par ailleurs, 4 communications viennent d'Allemagne (2) et d'Angleterre (2). Les villes françaises ne sont pas le seul centre d'intérêt : on trouve 4 études sur l'Allemagne, 3 sur l'Italie, 1 sur l'Angleterre, 1 sur le Portugal, 1 sur Marco Polo qui nous informe à la fois sur ce dernier et sur la Chine. D'une façon générale, l'histoire sociale est, avec 11 communications, la mieux partagée. Le paysage urbain et la réalité matérielle des villes ne sont l'objet que de 2 communications. Seul H. Dubois, de Paris-IV, présente une étude de démographie urbaine.

Plusieurs articles sont en même temps des publications de sources, œe qui devient rare. C. Vulliez, de Paris-X ( « Les étudiants dans la ville : l'hébergement des scolares à Orléans au bas Moyen Âge », p. 25-35), publie un contrat de pupillarité de 1442. W. Paravicini, de Kiel ("Charles le Téméraire à Tours», p. 47.69), publie l'itinéraire du comte de Charolais pendant son voyage en Bourgogne et à Tours du 30 septembre au 24 décembre 1461. M. Jones, de Nottingham ( $₫$ Nantes au début de la guerre civile en Bretagne ", p. 105-120), publie des lettres du " capitaine pour le Roy Monseigneur ez parties de Bretaigne " et de l'évêque de Nantes, en date du 11 mars 1342, sur la vente de pain, de viandes et de boisson, et les gages des ouvriers à Nantes. A. Vauchez, de Paris-X ( $₫$ Dévotion et vie quotidienne à Périgueux au temps de Charles $V$, d'après un recueil de miracles de Charles de Blois ", p. 305-314), publie un petit recueil de miracles des années 1370-1372 concernant la ville et la région de Périgueux qui est conservé aux archives du Vatican. J.-M. Vaccaro, de Tours ( «e croy en Dieu : la polyphonie de Pierre Certon pour les Articles de la Foy de Clément Marot \$, p. 393-399), publie le fac-similé de la transcription, conservée à la bibliothèque de l'université d'Uppsala, de la mise en musique par Certon de la traduction du Credo par Marot.

Le texte le plus inattendu de l'ouvrage est sans conteste celui, passionnant, de C. Deluz, de Tours ("Villes et organisation de l'espace : la Chine de Marco Polo », p. 161-168), qui dégage l'originalité extrême de la présentation de l'empire du Grand Khan que fait Marco Polo. « Dans l'espace politique ainsi défini, ce sont les cités qui rythment la progression de l'ouvrage [...] Au total 70 villes sont ainsi nommées par Marco Polo et ce qu'il en dit suit une sorte de plan type. " La description est explicative et "ce n'est pas une énumération de cités tout au long d'un itinéraire que nous donne Marco Polo, c'est un réseau urbain hiérarchisé qu'il met en place ". Cet « exemple brillant mais isolé " qui « est, en partie du moins, le témoin d'un apport de la géographie chinoise à la géographie occidentale " va nous servir de guide dans les textes ici rassemblés. En effet, C. Deluz dégage des descriptions du Vénitien la conception implicite de la ville qui les sous-tend : une ville c'est un statut, une histoire, un environnement, des activités spécifiques, un art de vivre enfin. Sur chacun de ces cinq aspects de la réalité urbaine, les "Mélanges Chevalier" apportent quelque chose.

Le statut de la ville la distingue du plat pays. Pour R. Fossier, de Paris-I, "Franchises rurales, franchises urbaines, dans le nord de la France » (p. 179-192) sont évidemment différentes mais les soucis qui furent à leur origine les rap- 
prochent (la paix, la protection des hommes et des biens, l'argent). Ces franchises sur lesquelles $M$. Fossier donne une belle synthèse sont l'aboutissement d'une lente évolution. Pour J.-M. Martin, du C.N.R.S. ( " Les communes en Italie méridionale aux $\mathrm{XII}^{\mathrm{e}}$ et $\mathrm{XIII}^{\mathrm{e}}$ siècles ", p. 201-210), si les communes sont une rareté dans le midi italien, le poids de l'histoire du haut Moyen Âge l'explique autant que la force de la centralisation monarchique : la "maturation » (R. Fossier) qui s'est produite dans le nord n'a pas eu lieu ici. Au reste, l'existence d'institutions municipales ne prouve pas en soi l'aboutissement d'un désir d'affranchissement : A. Chédeville, de Rennes-II, démontre ( La charte de franchises de Chartres, 1297 , et sa place dans l'histoire urbaine », p. 193-200) que les bourgeois qui négocièrent la charte de Chartres " craignaient les nouvelles formes et méthodes de gouvernement » et que la charte " apparait surtout comme une tentative pour freiner l'évolution ". Bref, le statut est nécessaire mais pas suffisant : cela pose le problème des petites villes. M. Pacaut, de Lyon-II, qui note qu'elles ont été moins étudiées que les autres, comble une lacune en étudiant la « Naissance et renaissance d'une petite ville : Louhans du XIII ${ }^{\mathrm{e}}$ au Xvie siècle » (p. 123-132).

Une ville, c'est aussi une histoire. Pour Gérald Chaix, de Tours (« L'historiographie colonaise à la fin du Moyen Âge », p. 315-322), si les chroniques urbaines se multiplient en Allemagne à la fin du Moyen Âge c'est parce que les villes allemandes, qui sont une forte réalité politique, «trouvent dans l'écriture de leur histoire un moyen de préserver leur identité " menacée par la puissance grandissante des États territoriaux. La comparaison avec les villes françaises, qui réagissent différemment et, pour M. Chaix, "perdent progressivement leur âme », est ici instructive.

Il n'y a pas de ville sans un environnement avec lequel elle vit en symbiose. Cela inclut la relation des hommes avec les animaux qui vivent en ville ou y passent, sur laquelle R. Delort, de Paris-VIII (« Les animaux dans la ville occidentale à la fin du Moyen Âge ", p. 343-350), fait le point des connaissances actuelles ; une synthèse sur ce sujet n'est pas possible avant plusieurs années. On connaîtra mieux la façon dont les villes tirent de la campagne ce qui leur est nécessaire avec l'article d'O. Redon, de Paris-VIII (« Une forêt urbaine : la Selva del lago », p. 247-257), qui analyse l'exploitation par la ville de Sienne d'une forêt communale qui arrivait près de l'agglomération et lui fournissait le bois nécessaire au chauffage. De même c'est la prise en compte de l'espace dans lequel s'insère la ville qui permet à F. Rapp, de Strasbourg-II ( «L'essor des villes et la multiplication des couvents au XIII ${ }^{e}$ siècle : les Mendiants dans la vallée du Rhin entre Bâle et Spire ", p. 289-296), de comprendre pourquoi il y a dans l'espace qu'il étudie près de 70 villes mais seulement 33 couvents de Mendiants installés dans 17 villes : les aumônes qui permettent aux frères de vivre sont reçues à la campagne aussi bien qu'en ville et la multiplication des couvents trouve sa limite dans le rétrécissement des zones de quête qu'elle entraîne.

On en vient aux activités et à la société proprement urbaines. Sur les activités économiques, les minutes des notaires apparaissent toujours irremplaçables : elles fournissent le matériau de 5 des 6 études d'histoire économique rassemblées ici. Ce sont des actes notariés, par exemple, qui permettent à P. Contamine, de Paris-IV (« Les gens de guerre et la ville : achat d'armures à Orléans 1434-1438 », p. 3-11), de trouver dans le commerce des armures d'Italie avant Jacques Cour 
des prédécesseurs de ce dernier. Sur les activités administratives, il faut se méfier des généralités admises : B. Guenée, de Paris-I ( ( Paris et la cour du roi de France au XIv siècle ", p. 259-265), démontre que c'est à la cour, et non à la présence des services du palais royal, que Paris doit une grande part de sa prospérité au xiv" siècle; "seules les structures économiques de la ville assurent la vie de la cour, et seule la présence toute proche de la cour garantit la prospérité de la ville $"$. Cette prospérité favorise le développement d'une criminalité économique (le vol) qui est au temps des Marmousets l'objet d'une attention particulière des services de la prévôté de Paris, parce que, comme l'explique C. Gauvard, de Paris-I, sa répression fait partie de la "réformation" (" La criminalité parisienne à la fin du Moyen Âge : une criminalité ordinaire ? », p. 361-370). La ville est aussi un lieu d'élection du crime politique par excellence, la trahison. L'affaire de ce type dont J. Tricard, d'Orléans, présente une analyse extrêmement fouillée ( « Une ville et son traître : Limoges et l'affaire Gaultier Pradeau, $x v^{e}-x^{e}$ siècle ", p. 211-222) montre que derrière le crime, acte individuel, il y a le jeu de partis dans la ville, et que derrière les divisions nées de la lutte pour le pouvoir il y a des divisions sociales dans la bourgeoisie : " les plus anciennes familles défendent avec acharnement les privilèges urbains. Les hommes nouveaux, suspects aux précédents, vont chercher fortune, au péril de leur vie, dans le parti adverse. " On est ici au çeur même de l'histoire sociale, car on ne comprend pas une société si l'on ne sait ce qu'il en est du pouvoir en son sein, et les études comme celle de M. Tricard ne seront jamais assez nombreuses. Enfin, en ville, on vit et on meurt; $H$. Dubois, de Paris-IV ( ¿ Démographie urbaine médiévale : trois modèles de mortalité à Dijon 1385-1407 », p. 333-342), présente des résultats de l'étude manuelle, préparatoire à l'étude informatique, de comptes de l'impôt dit des « marcs » de Dijon. Ces résultats, qui portent sur deux années de calme démographique et une année de peste, sont alléchants : $M$. Dubois est en mesure de calculer des taux de mortalité de la population masculine d'áge adulte $(29,9 /$ mille en $1386,173 /$ mille en 1401, 23,5/ mille en 1407), de chiffrer la surmortalité du temps de peste, d'affirmer que le rapport entre mortalité et pauvreté n'est pas établi, d'apprécier le dynamisme démographique - «il est mort $»$ après l'épidémie de 1399-1402 alors qu'il était élevé en 1385-1386.

La ville secrète un art de vivre et une culture. De cette culture font partie la perception et la construction par les hommes du temps, sur lesquelles J. Le Goff, de l'E.H.E.S.S., fait le point ( « La ville médiévale et le temps ", p. 325-332): le temps urbain est d'abord celui đe l'Église mais il se diversifie en assumant des fonctions nouvelles qui répondent aux besoins spécifiquement citadins. Sur la sociabilité urbaine, toutes les communications du volume sont instructives. J. Lafond, de Tours, l'ètudie spécifiquement (« Une vertu pour la ville : l'urbanité ", p. 401-410) et, à partir de La Conversation des Romains de Guez de Balzac (1644), montre que la diffusion au xvir siècle, dans la bourgeoisie, du salon et de la culture qui s'y rattache se comprend en scrutant les salons qui s'ouvrent dans la première moitié du Xviı siècle.

On n'a donné ici qu'un aperçu, impressionniste, injuste aussi - car on ne peut tout citer - de l'apport des « Mélanges Chevalier ». Il faut lire ce livre qui fait réellement honneur à celui auquel il a été offert. Il est une contribution de poids à 
notre connaissance de l'histoire urbaine et il offre un panorama des centres d'intérêt actuels de la recherche.

Thierry Dutour.

Jürgen KockA, Les Employés en Allemagne, 1850-1980. Histoire d'un groupe social. Trad. de l'allemand par Gérard Gabert. Paris, Éd. de l'École des hautes études en sciences sociales, $1989.15 \times 22,5,220 \mathrm{p}$.

Ce recueil d'études des années 1970, publié en R.F.A. dans une version revue et corrigée en 1981, n'est pas simplement, comme l'indique le sous-titre de l'édition française, l'histoire d'un groupe social du $\mathrm{II}^{e}$ au III' Reich - l'évolution après 1945 n'est traitee que dans les trente dernières pages. Emanant de l'un des spécialistes reconnus de l'" École de Bielefeld " à laquelle on doit aussi l'excellente revue Geschichte und Gesellschaft, il éclaire en effet un aspect de cette «voie spécifique » (Sondenweg) de l'histoire allemande qui expliquerait pourquoi, contrairement à d'autres pays capitalistes, l'Allemagne a basculé dans le nationalsocialisme. Cette question fut d'ailleurs débattue dès le début des années soixante entre historiens de la République fédérale d'Allemagne.

L'analyse menée " dans une perspective internationale, sans pour autant suivre une démarche comparative " (p.6) démontre la persistance d'un clivage particulièrement important entre employés et ouvriers, "l'existence dans les structures sociales allemandes de particularismes qui se sont révélés autant d'obstacles à la démocratisation de la société et de l'État " (p. 10). Évoquant successivement le statut des employés d'industrie du deuxième tiers du $\mathrm{xux}^{\mathrm{e}}$ siècle dans quelques grandes entreprises - en l'absence d'étude d'ensemble sur le sujet - l'émergence de la bureaucratie industrielle, les inégalités sociales sous le $\mathrm{II}^{\mathrm{e}}$ Reich, les évolutions politiques et sociales entre 1914 et 1933, les phénomènes de continuité et de discontinuité du III $^{\mathrm{e}}$ Reich à nos jours, cette étude rigoureuse, que complètent des statistiques, suit l'évolution de la «nouvelle classe moyenne » (neuer Mittelstand) dont lidentité se fonde sur une perception très hiérarchisée de la société et la peur de la déchéance sociale, qui entrave tout rapprochement avec le monde ouvrier. Situation que confortent les grands groupes financiers et politiques conservateurs en maintenant un statut spécifique hérité de l'ère préindustrielle, contraire à la rationalisation de la production et de la gestion mais qui permet de bloquer l'émergence d'un mouvement uni des salariés.

L'aspect le plus original de ce recueil réside dans l'analyse du glissement sémantique du terme Beamter emprunté vraisemblablement au latgage militaire des $\mathrm{xv}^{E}$ et $\mathrm{xv}^{e}$ siècles pour désigner d'abord les fonctionnaires titulaires, étendu, dans la seconde moitié du XIX ${ }^{\mathrm{E}}$ siècle au personnel des entreprises jouissant d'un statut comparable puis restreint, à partir de la crise économique de 1873, aux cadres et techniciens supérieurs ainsi qu'aux employés jugés méritants par leurs employeurs. À défaut du maintien d'un alignement général sur le statut de la fonction publique, les employés, soutenus par les groupes de pression conserva- 
teurs, obtiendront le maintien d'un statut distinct de celui des ouvriers et l'appellation spécifique d'Angestellte apparue au tournant du siècle et légitimée dans le cadre d'un régime particulier d'assurances (AVG) voté en 1911. En dépit des mutations socio-politiques, la République de Weimar maintiendra cette spécificité pour toute personne assujettie à l'AVG. L'infléchissement des organisations professionnelles d'employés vers un syndicalisme de gauche, au lendemain de la défaite de 1918 et de la faillite de l'Empire, n'est qu'un épisode de courte durée. J. Kocka admet certes que les études électorales des années 80 - notamment celles de Jürgen W. Falter, Hänisch, Hamilton et Prinz - atténuent quelque peu son constat d'une surreprésentation des employés dans l'électorat nazi des années 1930-1932. Il n'en démontre pas moins le glissement politique des employés déçus par l'évolution des années 1920 vers les forces représentatives des classes moyennes attachées aux traditions nationales, voire nationalistes et racistes. Attitude qu'il attribue à l'idéologie de leurs organisations professionnelles đont une minorité préconise le modèle corporatiste tandis qu'une majorité d'entre elles enferme ses membres dans l'ancienne conception d'un corps social distinct, par sa culture et son mode de vie, aussi bien de la couche inférieure du prolétariat que de la couche supérieure du capital. Sans ignorer l'interprétation marxiste de la lutte de classes, l'auteur insiste à juste titre sur le poids des traditions socio-culturelles, qui induit un clivage plus important entre ouvriers et employés en Allemagne que dans un "pays neuf" comme les U.S.A. Ce n'est qu'avec le III ${ }^{\mathbb{e}}$ Reich qu'intervient un début de nivellement imposé par le régime au nom de l'unité de "la communauté du peuple " (Volksgemeinschaft), qui se poursuivra après 1945 en République fédérale d'Allemagne par suite du rapprochement des rémunérations et du mode de vie des deux groupes sociaux.

Bien que des différences subsistent encore dans le recrutement, le niveau d'éducation et de formation, l'existence de collèges distincts ouvriers/employés dans les comités de gestion des entreprises et des caisses d'assurance, la situation paraît aujourd'hui comparable à celle des autres pays industrialisés en raison de l'effacement progressif du modèle d'identification et d'action corporatiste, de l'intégration croissante de la classe ouvrière et de l'atténuation des conflits d'intérêts entre les deux groupes.

Bien que J. Kocka devance la critique dans son avant-propos à l'édition française, on regrette ici l'absence d'un développement sur la culture et le mode de vie des employés, qui ont déterminé la spécificité de ce groupe social en Allemagne, et d'une analyse de la différenciation en son sein en fonction des sexes. L'ouvrage est utilement complété par une bibliographie raisonnée, qui comporte notamment des études facilitant la comparaison avec d'autres pays. La liste des abréviations d'institutions et d'organisations citées, assortie d'une traduction en français, compense quelque peu le manque d'index matières plus indispensable en roccurrence que celui des rares personnes mentionnées. Enfin, il faut souligner la qualité, assez rare dans $\propto$ type d'ouvrages, de la traduction qui permet au lecteur français, même non spécialiste, de suivre sans difficulté un travail que l'auteur qualifie de « très allemand».

Rita R. Thalmann. 
Michel Margairaz, L'État, les finances et l'économie. Histoire d'une conversion, 1932-1952. I et II. Préf. de François Bloch-LaiNé. Paris, Comité pour l'histoire économique et financière de la France, 1991. $16 \times 22,717 \mathrm{p}$. et $739 \mathrm{p}$. (" Histoire économique et financière de la France. Études générales ").

Big themes often justify big books and the theme here is an important one, the nature and causes of the changes in the French state between the Great Depression of 1929-1932 and the end of the post-war reconstruction. These changes, M. Margairaz argues, were a gradual uneven " conversion ", the word emphasized in his title, from a deflationary financial orthodoxy to more reflationary, demandled, economic and financial policies, representing a transition to a state founded on a broader social and political consensus. The method chosen to explain these changes, an intricately detailed historical narrative, is appropriate, he argues, because this was no sudden conversion, but a slow imperfect change of ideas, a fluctuating process whose ebbs and flows filled the whole period from 1935 to the end of his massive book.

Even by 1952 the new faith was insecurely held; cuts in public expenditure in that year would have been at the expense of the Monnet Plan's basic sector investment targets had the Planning Commissariat not been able to argue by that time that to cut these investments would have been more expensive than to continue them. The administrative structure of the state was little changed; the Ministry of Finance by 1952 had resolutely reasserted its primacy over the economic bureaucracy and attempts to create a separate ministerial economic bureaucracy concerned with production had come to nothing. If the mentality of high-level bureaucrats had changed that was partly an accident of war; their origins, recruitment and training had changed much less. Indeed, if the essential characteristic of a reflationary policy is a high ratio of government expenditure to revenue, France was more reflationary between 1935 and 1939 than after the war. The process which M. Margairaz wished to explain was thus neither chronologically straightforward nor self-evident, perhaps not even decisive-

The book is by far the most comprehensive history of economic policy formulation in France over the period. It is by no means confined to the operations of the Ministry of Finance. It contains also a comprehensive history of the installation and administration of the Monnet Plan, of Franco-American relations during the Marshall Plan, and of the economic relationship of the Vichy government to the German occupiers. The narrative is constructed from an exhaustive inspection of all available archives in France for the whole period. It is directed primarily towards illustrating three aspects of the conversion : changes in fiscal policy and in government expenditure; changes in state administrative structures; and changes in the ideas of politicians and bureaucrats. How well does this narrative method succeed?

The process of change which is described was not of course peculiar to France, it is also the story of the creation of the post-war state almost everywhere in western Europe. Outside the Iberian peninsula and Ireland all western European states were refounded after 1945 on the basis of a broader political consensus than the narrow and inadequate foundations which had led in many cases to their col- 
lapse from 1938 onwards. Ultimately, the political purpose of the state's attempt to alter and regulate the workings of the economy was to secure its political base by guaranteeing a greater and more secure income to large numbers of its citizens. In this way it rebuilt the allegiances which it had tended after 1929 to lose.

Part of this process of rebuilding allegiance is made clearer by the fine detail of M. Margairaz's narrative. Politicians like René Pleven or bureaucrats like Hervé Alphand in exile in London became interested in the ability of the state to maintain a higher level of employment and some, like Robert Marjolin, were strongly and directly influenced by Keynesian macroeconomics. So too were many of those in the Résistance. It was in discussions after 1940 over the way to resurrect France that the general implicitly Keynesian tendency of post-war policy became established and with it a different orthodoxy about the consequences of government expenditure. To some extent such policies were easier after the war. Reflation in the 1930s had always been impeded by an insufficiency of revenue. After the war, as before it, a distinguishing characteristic of the French economy remained the comparatively low level of state revenue relative to expenditure. The war and inflation, however, wiped out much of the state's previous debt, while American aid made a significant contribution to revenue in 1949 (13\%) and 1950 (8\%). But inflation and wartime debt conversions also carried the penalty that savings were less available for investment. The high investment ratios of the first three years after the war were financed by rediscounting medium-term loans through the central bank. The political will had changed during the war and the barriers of the 1930s were no longer seen as insurmountable.

Even though there was no agreement about the international economic framework for expansion, there was nevertheless after 1945 a political consensus, which Monnet encapsulated in his various activities, in favour of modernization, industrialization, and state intervention in the investment process until such time as the financial markets could take over. This was also a consensus against maintaining the dirigiste controls of Vichy. The biggest part in dismantling the Vichy government's control over resource allocation was played by a communist minister and until the Marshall Plan almost the whole of the left and centre can be seen from Margairaz's narrative to have gathered behind the Keynesian recipe for rescuing capitalism. Those few who did not, Andre Philip and Pierre Tanguy-Prigent with their desire for a central plan with a more dirigiste apparatus, Pierre Mendès France with his demands for cuts in consumption, austerity à l'anglaise, were the political losers. Theirs was not the way to the broader post-war consensus and the rescue of the state. It needed the detail in this book to make these points convincingly.

Other parts of the process of rebuilding allegiance, however, are only made more obscure by the narrative method. The most numerous single group of beneficiaries of the reconstructed state was in the agricultural sector. Occasionally politicians appear in M. Margairaz's pages arguing that agricultural prices should go up or down, but they always do so as part of a wider economic argument. The sudden increases in state investment in agriculture in 1948 are explained as a reaction to the dollar shortage and the Marshall Plan; Monnet, he implies, included agriculture as a basic sector in the revised Plan for balance of payments reasons. But comparison with any general European model of the post-war state would have 
shown that policy towards the agricultural sector closely resembled that elsewhere in Europe. A durable post-war consensus required agricultural income support. Poor Monnet! What choice did he have but to accept the concessions made by politicians to agriculture between 1945 and 1948 and to make the best of them by belatedly including agriculture in his Plan as another basic sector of the economy and so at least try to direct some of this lavish state dole, whose real purpose was political survival, towards the sacred cause of "modemization".

Here we see the problems of the narrative method and the lack of a general model. This is a narrative of action by state bureaucrats, not of the political decision-making process which preceded it. Of the intense pressure of the corporate agricultural interest on the state nothing is said, because it always took place before the policy changes which are described. The same is true of the state's greatly increased commitment to social security over the period. This omission is perhaps even more serious, because it is the increasing size of state budgets, swollen by income from social security funds, which made Keynesian macroeconomic stabilization more feasible. The state, as a result of the welfare concessions made by politicians seeking to establish their post-war legitimacy, acquired a much larger financial masse de manouvre. It is astonishing that France, one of the most comprehensive welfare states in the world, has produced no history of its creation. Such a history would have in the first place to be a history of political parties and of the parliamentary process. This is not the history which M. Margairaz wanted to write, but the sad consequence is that a major aspect of the "conversion" remains unexplained.

For all its genuine scholarship and energy the book's basic weakness is that of much French historiography, its exclusive interest in France and the assumption of uniqueness where comparison would have been more revealing. What else might comparison with a general model of the European state in the same period have revealed ? Reflation, as M. Margairaz argues, effectively meant inflation from 1936 to 1952 with only the briefest periods of stabilization. But why was France more inflationary than societies pursuing similar political objectives and making similar political concessions? Why was the external value of the currency given so low a priority compared to the priorities in other countries? Why did a country with roughly ten million voters depending on agriculture for their incomes move more diffidently towards comprehensive agricultural income protection than countries where the agricultural sector was electorally less important? Why did the demand for increased welfare concentrate so much on keeping the working week so short and overtime pay as a consequence so high? Why did it concentrate more on pay and less on other benefits? Why was it that a man operating entirely outside the framework of parliamentary politics, Monnet, created the most plausible framework for an agreed economic policy in a democratic framework, when in every other reconstructed post-war westem European state only parliamentary politicians were in a position to do this ? In spite of $\mathbf{M}$. Margairaz's wholly admirable efforts there are still many unanswered questions about the "conversion" of the French state, unanswered in part because the historical method chosen did not allow them to be accurately specified.

Alan S. Milward. 
Jean-Pierre Hirsch, Les Deux rêves du commerce, entreprise et institution dans la région lilloise (I780-1860). Paris, Éd. de l'École des hautes études en sciences sociales, 1991. $16 \times 24,534$ p., bibliogr., index, tabl. et fig. (« Civilisations et sociétés $\%, 82$ ).

À partir d'un double constat : celui de la continuité des pratiques et des hommes d'une part, celui de l'irrésistible ascension du libéralisme comme discours dominant, d'autre part, J.-P. Hirsch en établit un autre : le Commerce entendons les acteurs - a souffert d'un besoin institutionnel inassouvi, refoulé même dans la mesure où les élites du milieu ne sont jamais parvenues à le faire émerger à la conscience au point de l'exprimer clairement et sous une autre forme que de refus ou de revendication ponctuels.

L'interrogation centrale du livre s'applique à cette relation malheureuse d'un milieu à ses institutions : comprendre ses attentes et les réponses qui lui sont offertes, et, au-delà, les conditions spécifiques d'exercice d'une activité. Car I'hypothèse centrale réside en ceci : le divorce entre l'État et le Commerce n'est pas lié à une conjoncture particulière, mais à l'équation des paramètres fondamentaux et contradictoires d'une position et d'une activité. Coincé entre la course au profit et la course au crédit, le commerce requiert simultanément l'insertion au sein d'un réseau de relations, un ancrage solide dans l'assurance d'une fortune et d'un réseau de solidarités et la souplesse, l'indépendance, la hardiesse nécessaires à la conquête de marchés et đe positions renforcées. Il a donc besoin de liberté et de règlements, d'autonomie et de protection, d'aventure et de garanties. Cette hypothèse est assortie d'un ensemble de reconsidérations radicales et roboratives : de la coupure révolutionnaire, d'abord, de son sens et de ses limites. Mise en question aussi de la figure de l'entrepreneur individuel, véritable arlésienne de l'ouvrage, et de la parabole de l'indomptable ascension d'une bourgeoisie avide de liberté et d'individualisme. Démystification, enfin, de l'idée d'un âge d'or du libéralisme, caractérisé par l'avènement de rapports nus entre acteurs encadrés par la seule loi, égalisatrice, du marché. Au croisement de ces différentes approches, la thèse du «retard français " comme la théorie du " trop d'état " se trouvent mises en cause, problématique devenue familière au fil de quelques publications récentes'.

La portée de l'interrogation initiale comme l'hypothèse "structurelle " qui la soutient justifient le cadre grossièrement séculaire retenu par l'auteur, qui balaye largement la période entre 1780 et 1860 et ne s'interdit pas quelques incursions en deçà et au-delà. Des anciennes structures enracinées dans le privilège au réaménagement - provisoire - d'un nouvel équilibre libre-échangiste et libéral, il développe l'a analyse de quelques relations, quelques expériences choisies en petit nombre mais étudiées dans une durée suffisante pour en approcher le sens $»$. Quelques expériences? Mode d'exposition plus que dispositif de recherche:

1. Voir, en part., cité đ'ailleurs par Jean-Pierre Hirsch, Patrick Fridenson, André Strauss, eds, Le Capitalisme francais : 19 -20 siecle. Blocages et dynamismes d'une croissance, Paris, Fayard, 1987. 
l'impressionnante addition des sources imprimées et archivistiques prouve assez l'énorme travail érudit accompli pour traquer les indicateurs ou les indices pertinents susceptibles d'apporter des éléments de réponse ou d'étayer les interprétations. Signalons, par exemple, l'investigation systématique du fonds des faillites qui permet simultanément d'en dresser un bilan et de renouveler les perspectives sur l'événement, redéfini comme un instrument parmi d'autres de régulation et de liquidation des créances. Mais expériences circonscrites à une sphère sociale, à une région. Étudiant les relations d'un milieu à ses institutions, d'une communauté d'intérêts à l'État, il se donne les moyens d'en appréhender les représentations, les aspirations, non pas détachées de l'environnement qui les produit mais rapportées, réinsérées dans les pratiques des acteurs, saisis dans leurs différents champs d'intervention, domestique, professionnel, public (Chambres de commerce et différentes instances où ils évoluent). Le renfort d'une bibliographie étendue, notamment pour l'Angleterre, autorise des ouvertures et des comparaisons éclairantes avec l'espace retenu. Ainsi conçu, Lille et ses annexes roubaisienne et turquennoise, centre manufacturier d'un exceptionnel dynamisme, constitue plus que le cadre d'une monographie exemplaire promise par extension à une généralisation discutable : la compréhension de sa spécificité et de sa position relative oblige, selon les propos de l'auteur, à modifier l'idée qu'on se faisait jusque-là de la construction tout entière, c'est-à-dire de la croissance et du libéralisme français.

Le milieu auquel il s'attache, celui du " Commerce ", désigne avant la Révolution et pour longtemps encore, tant pour le dictionnaire que dans la réalité socioéconomique, tout à la fois le négoce, vendeur de tout et faiseur de rien, largement ouvert sur l'espace national et international, et le monde des fabricants, parfois encore tout proche de celui des métiers. Les deux univers sont étroitement unis, tant par des relations familiales que professionnelles. Ce milieu dirigeant, anciennement implanté - quatre ou cinq cents personnes -, s'est peu élargi au $\mathrm{XIX}^{e}$ siècle : il compte alors un millier d'entrepreneurs environ, parmi lesquels l'élite de la grande industrie, toujours indissolublement liée au négoce, est alors mieux représentée.

À la fin de l'Ancien Régime, les conditions - institutionnelles, sociales d'exercice de son activité placent cette micro-société dans un équilibre précaire, dont témoigne la multiplication du nombre des faillites, et lui laissent l'amertume d'une conscience malheureuse. Trois modes de gestion ou de réponse à ses besoins spécifiques sont alors à l'œuvre, sur des terrains distincts. Le terrain familial, où des stratégies matrimoniales habiles, usant de toutes les possibilités contractuelles offertes par le droit coutumier, permettent d'associer des fortunes, afin de former une communauté prospère, même en cas de rupture précoce du ménage, tout en sauvegardant les droits du lignage, limitant les risques en cas de faillite par exemple. Plus que la réunion de deux patrimoines, c'est toute une parentèle, toute une constellation de relations interpersonnelles qui se trouve agrégée, dans laquelle la maison ou la fabrique puisera ressources, informations, appuis, renommée et ouverture : "L'immersion dans le marché n'est pas tout, le succès dépend autant de la qualité des relations que du calcul économique. " Le cadre corporatif contribue également à réguler l'activité commerçante, au-delà de la sphère du privé. Faisant justice de la traditionnelle mais fallacieuse opposition 
entre capitalisme et corporations, négoce et métiers corporés, l'auteur montre au contraire comment le négoce s'appuya sur la corporation productrice de règles et de discipline, instance régulatrice des flux de main d'œuvre, des conflits de travail, des normes de production. Aussi la réappropriation du discours anti-corporatif apparaitt-elle davantage le fruit du réaménagement d'un argumentaire en cas de conflit avec le monde remuant des métiers urbains que celui d'une réflexion pénétrante sur leurs intérêts profonds. Au demeurant, insatisfait et fragilisé par la crise qui l'affecte, le monde du commerce se montre particulièrement inapte à formuler clairement ses attentes et ses besoins institutionnels, notamment en ce qui concerne le règlement des faillites. La justice du roi, interférant avec les décisions des tribunaux consulaires, est de son côté ressentie comme laxiste. En attente d'une législation commerciale unifiée qui leur garantisse la régularité et la transparence de leurs opérations, les entrepreneurs font plutôt bon accueil au plan intermédiaire conçu par Necker en 1778, habile à combiner la représentation du milieu et l'autorité supérieure, ménageant un équilibre inédit entre liberté et réglementation, aussi indispensables l'une que l'autre à la confiance et aux affaires. Mais cantonnés dans une position culturelle marginale et subordonnée, incertains des positions acquises et peu assurés de leur dignité, ils furent impuissants à faire entendre réellement leur voix.

Ils ne le furent pas davantage pendant la Révolution, qui ne leur apporta pas la stabilité institutionnelle espérée. Temps fort d'une évolution séculaire, celle-ci, loin de consacrer l'ascension d'une classe conquérante, acheva de consommer le divorce entre l'État et le Commerce.

En effet, on ne saurait trop souligner la gravité de l'échec de la tentative de " crédit national " inaugurée dès la Constituante, et qui discrédita aux yeux de ce milieu la figure du négociant citoyen. Ralliés massivement au projet constitutionnel - sans en être toutefois les promoteurs ou les soutiens d'avant-garde - ils réinvestissent dans celui-ci les valeurs de confiance, de réciprocité et d'honneur qui sont les leurs. La nouvelle donne socio-politique semble promettre les perspectives auxquelles ils aspirent confusément : autorité, citoyenneté, unité. Satisfaits du recul des barrières ou de la création des tribunaux de commerce qui leur ménage une place prépondérante, l'effondrement de tout l'édifice réglementaire dans la foulée de l'abolition des corporations, événement fondateur autant qu'improvisé, les plonge cependant dans un désarroi qui se révéla peu à peu. Par ailleurs, la mise en circulation des assignats, qui aurait pu sceller la réconciliation, tant enviée à l'Angleterre, du crédit public et du crédit privé, et offrir un formidable ballon d'oxygène à des entrepreneurs chroniquement gênés par l'étroitesse du marché des effets de commerce, n'apporta en fin de compte, passées les premières impressions favorables, que les déceptions et les embarras que l'on sait. Cette tentative avortée ne fit que dissuader un peu plus le monde des marchands de «se fourrer dans les affaires du Gouvernement ". Il faudra attendre plus de soixante ans avant que le marché des rentes d'État, enfin réintroduit sur la place lilloise sous la tutelle de la très officielle et très privilégiée Compagnie des agents de change, séduise les entrepreneurs libérés de la crainte du gouffre de la dette publique, et soit intégré au circuit de leurs propres valeurs. Même le Code de commerce (1807), pourtant inspiré par les pratiques anciennes et témoignant, par son caractère dérogatoire à la loi commune, d'une prise en compte de leurs 
besoins spécifiques, continua à nourrir le différend et la méfiance d'un milieu à l'égard d'institutions alternativement trop rigides ou lacunaires. Finalement, le discours surtout s'était déplacé. Mais en imposant la fiction d'une atomisation des rapports de production, en entretenant l'idée que le monde du négoce et de la fabrique s'organisait autour de rapports d'individu à individu, il avait obscurci les consciences et avait recouvert les problèmes - sans les résoudre - d'un emballage rhétorique dont les entrepreneurs, s'ils surent en user, ne souffrirent pas moins. Peu résignés à un repli sur soi et à une gestion séparée de leurs propres problèmes, ceux-ci, tout en cherchant à faire entendre leur voix auprès du gouvernement par l'intermédiaire des Chambres de commerce ou de l'Association de défense du Travail national, s'orientèrent sous le couvert d'un discours libéral, mais protectionniste, vers des solutions ou des instances de réglementation locales ou intermédiaires et s'appuyèrent sans réserve sur les bases traditionnelles de leur puissance : famille, patrimoine enrichi du joli pactol des biens nationaux, solidarités interpersonnelles. Ainsi, comment adhérer sans réserve à l'idée d'un âge d'or du libéralisme ou du triomphe de l'entreprise individuelle? Plus que jamais, celle-ci demeure une affaire collective, et l'individu n'est que l'élément d'un réseau. Plus que jamais les affaires requièrent confiance et régularité que la Loi, en l'état, est impuissante à assurer.

Dans le cadre manufacturier, fouetté par un dynamisme « à l'américaine ", le besoin de règlements, transcendant la pratique largement fondée sur la fiction de rapports purement contractuels, n'était pas moins grand. La police du travail, la lutte contre la fraude, la reconnaissance de normes de fabrication ne pouvaient résulter des seuls « arrangements entre soi », de mème que la définition d'orientations communes inévitables en cas de crise, ou l'élargissement d'un marché financier requis par des immobilisations croissantes en capital. Si les prudhommes, les municipalités ou l'usage avisé du livret pouvaient contribuer à instaurer des équilibres temporaires et locaux, l'entente et la définition claire d'intérêts communs eussent été hautement souhaitables.

Comment expliquer que les entrepteneurs n'en soient jamais venus là, et rendre compte du malaise persistant constamment refoule dans le non-dit? Jean-Pierre Hirsch esquisse plus qu'il ne les approfondit deux pistes interprétatives séduisantes : la première met en relief la portée de la négation de la notion de corps intermédiaires consubstantielle à la sacralisation de la volonté générale. Dans la construction socio-politique dessinée dès 1791 disparaît toute instance de dialogue intermédjaire dont le livre tend ả nous démontrer l'évidente nécessité pour un milieu caractérisé avant tout par un ensemble de pratiques et d'intêrêts communs. Mais eussent-elles existé, est-il sür que ces instances auraient été résolument réappropriées par les négociants et les manufacturiers? La peut sociale en eut de toute façon obscurci l'usage. Accepter le dialogue des intérêts communs et l'arbitrage de l'État, c'était admettre l'existence et l'expression collective d'un interlocuteur mystérieux et redouté : le monde du travail. C'était courit le risque que la coalition réponde au groupement d'intérêt, c'était affronter la redoutable "question sociale " à laquelle le milieu du commerce répondit au mieux par la mauvaise conscience et l'engagement charitable, jamais par la prise de conscience de l'impératif d'un traitement collectif et politique. Les lecteurs regretteront certainement que cette thèse ne soit pas davantage développée : l'auteur retrvoie sur 
ce point aux pages éclairantes rédigées pour le tome trois de l'Histoire de la France parue au Seuil ${ }^{2}$. De la même façon, on aurait aimé que prît davantage d'ampleur l'analyse du moment décisif de 1791 où se cristallisa la rupture entre deux logiques, celle, politique, de la Nation, et celle d'un milieu qui ne parvint jamais dès lors à se penser comme une addition d'individualités ni à assumer pleinement sa dimension collective.

De cette lecture, rendue difficile par cela même qui la rend attrayante, l'extraordinaire foisonnement d'exemples minutieusement disséqués, autant d'îlots où le lecteur est exposé à une déviance métonymique, on retire la certitude qu'un certain nombre d'obstacles sont définitivement levés, et que le problème de la relation État-entrepreneurs est désormais posé en termes adéquats et féconds. Il n'en rend que plus aiguë l'interrogation sur ce qu'est, finalement, le libéralisme. Pur effet de discours? Ou dialogue pour faire coïncider une construction politique avec des configurations, des pratiques, des représentations qui lui échappent? En même temps que les " Deux rêves du Commerce ", Jean-Pierre Hirsch nous fait approcher les deux rêves de l'historien : donner à voir une société ou une époque "dans la familiarité essentielle à la compréhension des problèmes sociaux", et donner à penser des problèmes qui sont plus que jamais d'actualité : la pertinence des réponses libérales et du discours de la concurrence aux défis des contradictions et des inégalités sociales comme des exigences de la croissance.

Dominique Margairaz.

\section{HISTOIRE DU SAVOIR ÉCONOMIQUE}

Ars Mercatoria. Eine analytische Bibliographie. Bd $1: 1470-1600$. Mit einer Einleitung in deutscher und französischer Sprache. Herausgegeben von Jochen Hoock und Pierre Jeannin. 6 Bände. Paderborn, Schöningh, 1991. 17,5 x 24,5, LIV-432 p.

La connaissance du monde des marchands est enrichie de la publication du premier tome des Ars Mercatoria, répertoire bibliographique des manuels et traités à l'usage des marchands, qui recense les ouvrages dont la première parution attestée est antérieure à 1601 , en attendant la livraison ultérieure d'un volume qui couvrira les années 1601-1820. Près de deux mille références, plus de milke auteurs repérés dans toute l'aire de civilisation européenne en dépit des conditions inégales d'investigation, signalés par un descriptif précis (auteur, titre intégral, éditeur, imprimeur, lieu de consultation ou source secondaire où se trouve mentionné l'ouvrage, codage analytique, etc.). Un index par auteurs, une chrono-

2. André Burguière, Jacques Revel, dir., Histoire de la France. 3. Les Conflits, Paris, Seuil, 1990. 